\title{
A Critical Review of Healing Elements: Efficacy and the Social Ecologies of Tibetan Medicine
}

\author{
Michelle Gowan* \\ Department of Archaeology and Anthropology, College of Arts and Science, University of Saskatchewan
}

\begin{abstract}
This article offers a critical review of Healing Elements: Efficacy and the Social Ecologies of Tibetan Medicine. The ethnography provides rich and comprehensive insights regarding the triumphs and tribulations of Sowa Rigpa (traditional Tibetan medicine) as the medical system is translated across diverse contexts to ensure its continuity within the globalized world; however, these insights can be broadened by more deliberately acknowledging and investigating the (post)colonial subtexts underlying these translations. Incommensurability emerges throughout the ethnography in the form of tensions that arise as tacit knowledge is translated to explicit knowledge in the quest for legitimization. It is argued that expounding the nature of this incommensurability by engaging with rather than rejecting polarized notions of "traditional" and "modern" paradigms can reveal that non-biomedical medical systems and medically pluralistic contexts more broadly are inundated by (post)colonial processes. Borrowing Blaser's (2013) notion of "Sameing," it is demonstrated that translation involves (post)colonial processes of assimilation, as Sowa Rigpa is rendered visible through Good Manufacturing Practices (GMP), and appropriation, as it is made palatable through pharmaceutical commodification. Furthermore, it is argued that these processes mobilize mimesis and essentialization to transform Sowa Rigpa into a system that is both legitimized and acquiescent to the imperatives of varying external regimes. The simultaneity of these effects and the position that they are not mutually exclusive is asserted throughout the review as further evidence of (post)colonization.
\end{abstract}

Keywords: critical review, Sowa Rigpa, traditional Tibetan medicine, efficacy, (post)colonialism, assimilation, appropriation

\section{OVERVIEW}

In Healing Elements: Efficacy and the Social Ecologies of Tibetan Medicine, Craig (2012) employs the following questions to guide the exploration of what it means to say that a medicine "works": "how is efficacy determined? [And] what is at stake in these determinations?" (4). These questions are considered within the context of translating and therefore legitimizing contemporary Sowa Rigpa (Tibetan Medicine) as a valid medical system according to the standards of varying external regimes. Craig uses creative nonfiction to recount this multi-sited ethnography, which spans approximately one decade and draws on observations, exchanges, and

\footnotetext{
*mlg021@usask.ca
} 
reflections from sites in Nepal, Tibetan areas of China, Bhutan, and the United States. The overarching analysis considers the translations that take place through the engagements between the traditional Tibetan medical system and the varying imperatives imposed by modern external regimes and entities, including biomedicine, clinical research, national and international governmental regimes, commodification processes, conservation-development projects, and identity politics. These engagements occur as the proponents of Sowa Rigpa-including the Tibetan and Himalayan practitioners known as amchi, patients, and administrators navigate the incommensurability of traditional and modern paradigms.

Craig (2012) considers efficacy throughout these navigations using the theoretical framework of social ecologies, defined as "the interrelationships among environmental, socioeconomic, biological, political, and cosmological sources of, or explanations for, health problems" (5). This framework extends beyond acknowledging the relationship between health and environment. Instead, the approach conceptualizes nature as a system of processes and as a locus of sociocentric relations between human and non-human beings and the divine forces that contribute to an "animate earth" (Craig 2012, 6), a concept that reiterates the sentiments underlying Tibetan medicine. Throughout the ethnography, Craig demonstrates that legitimizing Sowa Rigpa relies on explicating the medical system's efficacy. This work involves translating Sowa Rigpa's traditional paradigm, which in practice depends on the translation of tacit knowledge; this is the central premise of the work.

\section{CRITIQUE}

The overarching theme of this ethnography is translation; namely, the translation of tacit knowledge to explicit knowledge. Tacit knowledge refers to "knowledge that is not explicated" (Collins 2010, 2; emphasis is my own) while explicit knowledge is that knowledge that is articulated. This translation is demanded by competing and conflicting evaluative frames imposed by the imperatives of the aforementioned external entities and regimes, which are used to determine the legitimacy of Sowa Rigpa. Tensions arise when considering the divergence between whether tacit knowledge "is not" or "cannot" (Collins 2010) be explicated. This tension is most pronounced in the demand for demonstrating efficacy in order to verify Sowa Rigpa's utility; a pressure which saturates this ethnographic context. This demand is based on an epistemological claim that reveals a predilection for one system of knowledge (that which undergirds biomedical science) over others (that which undergirds Tibetan medicine) (Coulter 2004). Sowa Rigpa proponents must cautiously navigate this predilection, as it incurs the "risk of irremediable loss or change of meaning" (Collins 2010, 26; emphasis by author). This risk refers to the epistemic violence that occurs when the process of demonstrating efficacy can cause treatment modalities to be "stripped of the paradigm within which they traditionally resided" (Coulter 2004, 114) and therefore subjugated and reduced.

While the nature of this incommensurability as an epistemological predilection that is born of a paradigmatic clash and perpetuated by (post)colonial underpinnings is not expounded explicitly in the ethnography, this review will assert that such elaborations can strengthen the work's broader relevance significantly. To clarify, the translation of tacit knowledge to explicit knowledge involves navigating incommensurable traditional and modern paradigms. This incommensurability manifests in the form of tensions, which Craig (2012) notes by recalling instances during translation (the translation of language, practices, and materials) when words "resist trans- 
lation" and objects "defy exchange" (252). But what does this mean? What is being defied and resisted? And why? Craig does address paradigmatic barriers by challenging and refusing to dignify views that entertain the limiting polarized notions of "traditional" (underdeveloped) versus "modern" (developed) medicine; however, in rejecting the premise of these polarities by refraining from engaging with them overtly, the (post)colonial mechanisms undergirding and sustaining them, as well as the subsequent incommensurability that ensues throughout the ethnography, remains relatively uncontextualized. The wider implications of this lack of contextualization are that it contributes to maintaining the covertness of (post)colonialism as it operates under the guise of modernity and, therefore, must be rectified if proper exposure and interventions are to occur.

\section{ANALYSIS}

Put simply, (post)colonialism refers toor rather, implies - the aftermath of colonialism. Despite the fallacy perpetuated by the term, this definition requires an elaboration on colonialism. Fundamentally, colonialism is a process perpetuated by the production of culture as a systematic and bounded entity, which is premised on the dualistic concepts of self and Other (Abu-Lughod 2006; Hall 1992). This opposition is enacted by "dismantling the essential categories of [O]ther societies" (Watts 2013, 31) and simultaneously reiterating the self. This distinction is sustained and operationalized by coercive power structures (Watts 2013; Blaser 2013), which reinforce a dominant self and, therefore, a dominated Other. Consequently, in considering colonialism as the production of culture and culture as the production of a self and an Other, (post)colonialism can then be understood as a shift in production processes - namely, a shift from Othering (understood here as characterizing colonialism) to Sameing (understood here as characterizing Blaser's [2013] notion of an "all-encompassing modernity" and therefore [post]colonialism). Borrowing Blaser's (2013) connotation of "Sameing," this critical analysis asserts that the translation of tacit knowledge involves what will henceforth be referred to as Sameing strategies, which are revealed to be (post)colonial processes underlying the legitimization of medical systems.

The notion of an all-encompassing modernity is discussed by Blaser (2013) as a process related to "European expansion and its effects" (549) (namely, colonialism), which "engulfs cultural differences" (ibid., 548). This discussion considers methods of Othering and Sameing as polarized means to the same end: the homogenization of the dominant self and the dominated Other. While Othering perpetuates the self/Other divide and relies more on coercion to achieve homogenization, Sameing collapses the self/Other divide and relies primarily on persuasion to achieve homogenization. In the wake of this "collapse," Blaser (2013) asks the question, "whose self becomes naturalized?" (549). Although a collapse may feign neutrality, the hegemony of the Eurocentric and paradigmatically modern self remains in tact; its dominance is secured and induced through the authority of a "universal science" that claims to be able to ascertain the "Truth", as per its many demonstrated feats since its inception in the sixteenth century (Blaser, 2013, 555). This persuasion serves to subjugate the Other in the image of the dominant self; in this context, the subjugation of Sowa Rigpa in the image of an ideology of science (Craig 2012). Craig uses this term to illuminate that the validity of traditional medical systems is based on their explicability and subsequent alignment with biomedical standards; this is the overarching mode of engagement through which traditional Tibetan Medicine is legitimized and with which it must coincide. 
Rather than directly engaging with polarized notions of traditional and modern paradigms, citing the limitations of a bounded dualism, Craig (2012) instead engages with and adopts the metaphor of a mosaic to emphasize the complexity and fluidity of this medically pluralistic context (9). However, this negation posits a neutrality that leaves the power dynamics that permeate this context unexposed and therefore unchallenged. Meanwhile, the imposing nature of the power-laden self/Other divide is particularly evident in the absence of this division in the social ecologies underlying Sowa Rigpa, wherein humans, nonhumans, and environment are all connected through overlapping capacities of intentionality and agency (Craig 2012; Povinelli 1995; Watts 2013). Subsequently, incommensurability (again, understood as the tension between traditional and modern paradigms) occurs as the (post)colonial structures, which underly external regimes and are premised by a self/Other divide, endeavor to evaluate these Other systems based on their truthfulness; in this case, their efficacy. This results in two forms of subjugation. First, these systems are "disqualified" (Foucault 1994, 203) as beliefs "rather than a method of ascertaining truth" (Povinelli 1995, 506). Second, these systems are "insufficiently elaborated" (Foucault 1994, 203) and distilled as "provocative or interesting interfaces of accessing the real" (Watts 2013, 26). To this end, in their response to "qualify" and "sufficiently elaborate" Tibetan medicine, Sowa Rigpa proponents must engage with concomitant processes of translation and legitimization aimed at validating the Tibetan medical system according to the biomedical regulations, policies, and standards delineated by an ideology of science (as well as the regimes that it informs). Rather than challenging views of polarized portrayals of "traditional" and "modern" categories by rejecting the premise of such notions, it may be more useful to instead expound the nature of the incommensurability that is born of the structures and processes that facilitate such polarities. When expounded, it is revealed that translation relies on approaching incommensurability by engaging in Sameing strategies, which are in effect (post)colonial processes of assimilation and appropriation. This analysis therefore illuminates the power-laden reality of medically pluralistic contexts and more accurately depicts the challenges faced by non-biomedical medical systems.

\section{(POST)COLONIAL PROCESSES; SAMEING STRATEGIES}

The struggle of Sowa Rigpa proponents to revitalize and validate their medical system takes place within a (post)colonial paradox of coerced/persuaded consent (Blaser 2013, 555) that demands the demonstration of efficacy. This paradox can be understood by revealing that the translation of tacit to explicit knowledge mobilizes Sameing strategies of assimilation and appropriation. These strategies are employed as Sowa Rigpa is translated through mimesis, in the form of assimilative Good Manufacturing Practices (GMP), and through essentialization, in the form of appropriative pharmaceutical commodification. Consequently, Sowa Rigpa is translated and transformed into a system that is legitimized and that is more readily acquiescent to the imperatives of varying external regimes, such as the Chinese government and the pharmaceutical industry. It is therefore argued that reiterating an "all-encompassing modernity" as both the collapse of the Eurocentric self/Other divide and the subsequent imposition of the homogenous category of efficacy in this context reveals that the translation of tacit knowledge to explicit knowledge to legitimize Sowa Rigpa is predicated on Sameing strategies of assimilation and appropriation and is therefore a (post)colonial endeavor. In other words, the possibility that Sowa Rigpa, and 
traditional or non-biomedical medical systems more broadly, are being (post)colonized must be more directly considered and investigated, particularly in the wake of globalism and the emergence of "integrative" medicine.

\section{Mimesis: Assimilation through Good Manu- facturing Processes (GMP)}

Knowledge systems can be weaponized against other systems by imposing "regimes of truth" (Foucault 1980) in order to derail the power of these other systems' legitimacy. As per the nature of oppression, there are a limited number of ways in which those systems experiencing assault can respond. One response is to invoke mimesis as a method to derive (or regain) legitimacy by reflecting aesthetic components to garner the validation that is deemed necessary. Before continuing, it is incumbent here to note the contentions surrounding the concept of mimesis, particularly because its use here falls directly along the axis of these disputes. Debates are particularly fervent following Taussig's applications, which Huggan (1998) critiques, and are typically regarding the function underlying the nature of the "mimicking" or "imitation" that might occur in contexts involving dominant and dominated groups ([post]colonial contexts); namely, whether imitation of the former by the latter is strategically performative or meant to genuinely mediate between systems (Huggan 1998, 94). Fully divulging the details of this debate is beyond the scope of this review but put simply, it reflects a question of whether the resemblances that occur when dominating and dominated systems meet are more superficial and external or more genuine and internal; of course, there remains the issue of how "superficial" and "genuine" are to be defined. Concurring with Craig's (2012) views on such polarities, it is likely neither one nor the other exclusively; however, this still presents a complicated question, as the answer will reflect the extent to which the agency of the dominated system can be exercised. Consequently, this question echoes a much broader discussion concerning the balance between "dark anthropology" (which focuses on the more difficult aspects of social life) and an "anthropology of the good" (which focuses on the more optimistic dimensions of social life) (Ortner 2016, 47). And while it is not im- possible to hypothesize about such things-indeed, this is often a primary component of an anthropologist's role as a mediator - it must be noted that declaring intent or outlining agency incorrectly can lead to either paternalistic conclusions that overstate strife and reduce agency or dismissive conclusions that reduce strife and overstate agency. That being said, although underlying intent will vary with context, the process of "imitation" remains rather standard and will be the focus of this discussion.

To reiterate, these complexities do not necessarily prohibit the utility of the concept of mimesis, rather they invite an opportunity for critical engagement and demand that the concept be applied mindfully, which is the ideal standard for all concept use. So, keeping its history in mind, the explanatory power of mimesis to consider the phenomena derived in Healing Elements will be delineated as clearly as possible moving forward. Ideally its application here will either contribute critically or be the object of critique in ways that will contribute to future elaborations of the concept and its use in (post)colonial contexts.

To continue, mimesis refers to "the capacity of people to see or create resemblances between themselves and others or to identify uncannily with their object of representation" (Huggan 1998, 93). This process is instigated by the power of accusation wherein other modes, or Others' modes (Hall 1992), are subdued by the structures of the dominant self and then induced to participate in their own assimilation through mimesis. To this end, assimilation occurs in the context of Sowa 
Rigpa legitimization, potentially as a topdown process that risks progressing from an aesthetic camouflage to engaging in "deconstructive ventriloquism" (Hall 1992, 286), whereby the regurgitation of biomedical principles and practices meant to legitimize Sowa Rigpa run the risk of eroding and replacing those that define the traditional system.

Again, while it is outside the capacity this review-and this reviewer, as such conclusions must be directly informed by those more directly experiencing a context - to determine whether this method is more helpful or more harmful, it is certainly present and emerges in Healing Elements through the introduction of Good Manufacturing Processes (GMP). These are strict regulatory conditions regarding the evaluation and processing of materia medica (medicinal materials, specifically plants) as defined by the "regimes of pharmaceutical governance" (Craig 2012, 24). GMP are introduced to ratify accusations of insufficient standards. Subsequently, they are implemented as a criteria for medicines, resulting in the enforced mimesis of factory production facilities. This includes factories fabricating the "look" of GMP in terms of architecture, which results in "spaces of incommensurability" (Craig 2012, 59), meaning production regulations that contradict traditional methods. An example of this contradiction occurs in what Craig (2012) notes as the "fetishization of cleanliness" $(163,201)$, which recasts flowers, some of which are used to make Tibetan medicines, as a form of pollution and requires that they be removed from the vicinity of medicinal production facilities. In this sense, GMP enhances visibility of Tibetan Medicine "while draining its force" (Huggan 1998, 99). Further issues arise when these regulatory apparatuses themselves become symbols of legitimacy, and GMP-certification becomes necessary in "rendering Tibetan Medicine legible within the context of contemporary China" (Craig 2012, 158).
Consequently, the legitimacy of Sowa Rigpa is qualified using mimesis to translate tacit knowledge through an operationalized assimilation to explicit knowledge. (As an aside, and for future consideration regarding the contention and complexity surrounding the concept of mimesis, perhaps the simplest way to approach this uncertainty is to first consider the question, "who is the operator?")

\section{Essentialization: Appropriation through Commodification}

Tibetan medicine, in the form of statecertified commodities, involves the commodification of a central element to Tibetan culture (Nigh 2002) - Sowa Rigpa materials. The process creates a product that is both "mystical and scientifically proven" (Craig 2012, 180); however, the latter paradoxically involves medicines being stripped of their paradigm of origin, followed by a romanticized version of this tacit knowledge then being fabricated as explicit. The commodification process therefore relies on the dissection of medicines; this operation includes the extraction of those parts that align with science and the rejection of those parts which do not. In this way, the elements that define Sowa Rigpa are either "derided as spurious or embraced as authentic" (Blaser 2013, 559), and done so according to the extent to which these elements "can be made to fit existing and interested preconceptions" (Ibid). Those aspects that are embraced refer to those which "allopathic medicine [and other regimes] can intellectually comprehend and commercialize" (Nigh 2002, 469). Next, the same colonial lens that performed the dissection is used to recall the context that it disassembled. Authenticity is then restored (though arguably, and ironically, manufactured) by a romanticized and "homogenizing gaze" (Nigh 2002, 452). Through this process, Tibetan culture is transformed and hypercontextualised into a 
static display. Subsequently, anxiety emerges that "perhaps the display is insufficient to prove authenticity [, and] [t]he culture of display is granted the status of a standard against which other instantiations of authentic identity can be gauged" (Hankins 2012, 14). In other words, this describes the process through which stereotypes (Hall 1992) are created and sustained. The resulting product represents "a Tibet that is culturally intact, spiritually infused, politically docile, and scientifically potent" (Craig 2012, 179). Tibetan medicine is therefore translated in this context by effectively appropriating tacit culture through (post)colonial essentialization in the form of commodification.

\section{DISCUSSION}

Craig's (2012) discussion of colonialism includes noting that in the context of this work it is not isolated to reiterating Western ambitions, but also includes the pressures of Chinese regimes. This consideration can be extended by articulating that the tensions discussed in Healing Elements are provoked by and arise from a need for justification of Tibetan medicine, which is necessitated by a (post)colonial context. Throughout the ethnography, Craig comprehensively notes the efforts of amchi to "secure and revitalize their practice in the face of major socioeconomic, cultural, and political change" (Craig 2012, 18); however, challenging polarized notions of "traditional" and "modern" by refusing to engage with them directly leaves the fact that these changes are implicated within a (post)colonial context relatively uninvestigated. Moreover, this leads to the dangerous possibility that the contradictions and compromise that result from these efforts will be regarded as necessary sacrifices in the quest for external legitimization rather than as casualties incurred through the subjugation of this traditional medicine.
Moreover, in declining to explicitly engage with and contextualize this dichotomy thusly, incommensurability may be misunderstood as a result of the presumed fixedness of traditional paradigms. The consequences of this omitted context are that tensions marking the aforementioned resistance (for example, of language) and defiance (for example, of objects) (Craig 2012) might appear instead as a noncompliance or defiance of tradition with modernity and are then susceptible to being explained away as the ignorance or uncertainty of the "fixed" former (Smithson 2012). These are dangerous reductions. Alternatively, by acknowledging incommensurability as a (post)colonially-perpetuated process, it is possible to expose these paradigmatic tensions as the result of the rigid and incessantly inflexible (post)colonial structures undergirding an all-encompassing modernity (Blaser 2013). To this end, it is then possible to challenge the contrasting and reductive connotation of tradition that denotes stagnancy, and instead to reveal the flexible and dynamic nature of this paradigm (and the entities derived from it, such as non-biomedical medical systems). For instance, defiance and resistance in response to the impositions of a modern paradigm whose (post)colonial underpinnings are acknowledged subsequently recasts these responses as protests against (post)colonial processes (Hall 1992). This distinction, which exposes the ironically consistent structures underlying modernity and reveals the overlooked dynamism of tradition, is what is at stake in these elaborations.

\section{CONCLUSION}

By emphasizing the centrality of translation in this work without outlining the underlying (post)colonial structures upon which this translation is grounded, these translations are susceptible to being misrepresented solely as negotiations between traditional and modern paradigms rather than as navigations of the 
power relations within which these maneuvers are implicated. This is an important distinction because the former denotes being subject to encounters with external regimes while the latter involves asserting agency though strategic engagement with these regimes. Craig's (2012) approach to erode this dichotomy by reiterating that amchi practitioners engage in developmental processes, such as empiricism, as a part of their practice is noteworthy; however, more directly acknowledging the structures underlying the translation of Tibetan Medicine enables the tensions that arise to be better understood beyond what might be presumed as a tired (though, never retired) clash between traditional and modern paradigms. Consequently, rather than appearing as a traditional system's incompatibility with modernity, these tensions can then be contextualized as the result of the imposed homogenous category of efficacy, which leads to assimilation and appropriation - the Sameing strategies - indicative of (post)colonialism.

Blaser (2013) articulates an important quandary, asking a question of "how to overcome this Sameing that transmutes the inherent hybridity of cultures into ethnographically 'thin' differences unified under the banner of modernity (be they defined as the capitalist world system or otherwise?" (549). It is offered here that contextualizing that the translation of tacit knowledge to explicit knowledge takes place in a landscape terraformed by (post)colonial processes would allow for a more accurate depiction of the dynamism, adaptability, overarching inventiveness, and therefore value of traditional paradigms, such as that which informs Sowa Rigpa. Neglecting to outwardly acknowledge the (post)colonial frame that undergirds the paradigmatic clash between dichotomized notions of "traditional" or "modern" systems leaves this scaffolding unpronounced, unchallenged, intact, and therefore enabled. While this contextualization may not overcome the realities which it describes, at the very least, it offers a recourse that is far less complicit than otherwise ignoring, and subsequently enabling, the nature of these (post)colonial processes.

\section{REFERENCES}

Abu-Loghod, Lila. 2006. "Writing Against Culture." In Feminist Anthropology: A Reader, edited by Ellen Lewin, 153-169. Boston: Blackwell Publishing.

Blaser, Mario. 2013. "Ontological Conflicts and the Stories of Peoples in Spite of Europe: Towards a Conversation on Political Ontology." Current Anthropology 54, no. 5 (October): 547-568. https://doi.org/ $10.1086 / 672270$

Collins, Harry. 2010. Tacit and Explicit Knowledge. Chicago: University of Chicago Press.

Coulter, Ian. 2004. "Integration and Paradigm Clash: The Practical Difficulties of Integrative Medicine." In Mainstreaming Complementary and Alternative Medicine, edited by Philip Tovey, Gary Easthope, and Jon Adams, 103-122. New York: Routledge.

Craig, Sienna R. 2012. Healing Elements: Efficacy and the Social Ecologies of Tibetan Medicine. California: University of California Press.

Hall, Stuart. 1992. "The West and the Rest: Discourse and Power." In Formations of Modernity: Understanding Modern Societies, an Introduction, Book 1, edited by Bram Gieben and Stuart Hall, 275-331. Cambridge: Polity Press. 
Hankins, Joseph D. 2012. "Maneuvers of Multiculturalism: International Representations of Minority Politics in Japan." Japanese Studies 32, no. 1: 1-19. https://doi.org/10.1080/10371397.2012.6 69730

Huggan, Graham. 1998. "(Post)Colonialism, Anthropology, and the Magic of Mimesis." Cultural Critique 38 (Winter): 91-106. https://doi.org/10.2307/1354379

Foucault, Michel. 1980. Power/Knowledge: Selected Interviews and Other Writings, 1972-1977. Edited by Colin Gordon. Translated by Colin Gordon, Leo Marshall, John Mepham, and Kate Soper. New York: Pantheon Books.

Foucault, Michel. 1994 [1976]. "Two Lectures." In Culture/Power/History: A Reader in Contemporary Social Theory, edited by Nicholas B. Dirks, Geoff Eley, and Sherry B. Ortner, 200-221. Princeton: Princeton University Press.
Nigh, Ronald. 2002. "Maya Medicine in the Biological Gaze: Bioprospecting Research as Herbal Fetishism." Current Anthropology 43, no. 3 (June): 451-477. https://doi.org/10.1086/339745

Ortner, Sherry B. 2016. "Dark Anthropology and its Others: Theory Since the Eighties." HAU: Journal of Ethnographic Theory 6, no. 1: 47-73.

Povinelli, Elizabeth A. 1995. "Do Rocks Listen? The Cultural Politics of Apprehending Australian Aboriginal Labor." American Anthropologist 97, no. 3 (September): $\quad 505-518$. https://doi.org/10.1525/aa.1995.97.3.02a 00090

Smithson, Michael. 2012. Ignorance and Uncertainty: Emerging Paradigms. New York: Springer-Verlag.

Watts, Vanessa. 2013. "Indigenous Place: Thought and Agency amongst Humans and Non-Humans (First Woman and Sky Woman Go on a European World Tour!)." Decolonization: Indigeneity, Education, \& Society 2, no. 1: 20-34. 